$\xi=-1$

\title{
Analysis technique of a wearable IoT health information on the MAPHIS
}

\author{
Kwang-Man Ko ${ }^{1}$, Soon-Gohn Kim $^{2} *$ \\ ${ }^{1}$ Dept. of Computer Science and Engineering, Sangji University, 26339, South KOREA \\ ${ }^{2}$ Dept. of Computer and Game Engineering, Joongbu University, 32713, South KOREA \\ *Corresponding author E-mail: sgkim@joongbu.ac.kr
}

\begin{abstract}
Background/Objectives: As u-health becomes common that monitors body condition real time in the ubiquitous environment, people are increasingly interested in promoting their health using biometric information identified by various health equipment.

Methods/Statistical analysis: the concept of digital health has emerged that encompasses the followings: $u$-health that is expected to improve efficiency in medical service and monitor patients' condition with wireless communication through convergence of ICT and health care industry; smart health(s-health) that manages their own workout, calorie intake and sporting activities with their smart device; and, mobile health (m-health) that uses wearable and mobile devices as a means of healthcare.

Findings: In this study, we aim to develop a health care platform that receives diabetes information generated from various IoT based on remote inputs, stores, analyzes, processes and provides visualized information. The purpose of this study is to develop and test IoT-based diabetes health big-data platform for diabetes mellitus patients. To achieve this goal, we suggest the development result of service and contents oriented "An IoT-based diabetes health big-data Offloading platform" that comprehensively manages healthcare products created in many IoT-based diabetes information to build a personal health management system. We also developed android 4.x-based application so that the health management service and contents provided by a third party can be checked with the client PC as well as health management service and contents offered by web-based client application and third party can be operated in the mobile environment such as smartphone or tablet.

Improvements/Applications: The results of this study are verified by applying it to patients with diabetes or suspected cases. In order to increase the efficiency of real-time processing, we used off-loading technology to utilize big data related to diabetes generated from wearable IoT device. The results of this study will be used for telemedicine in two hospitals in Malaysia after various laboratory verification procedures.
\end{abstract}

Keywords: IoT; Healthcare; Bigdata; Offloading; Diabetes.

\section{Introduction}

As the medical data-based precision medicine evolves, the medical data industry that generates, collects, and utilizes vast amounts of medical data is growing significantly. Research organizations and institutions that use medical data collect and analyze data around medical claims. Expert medical institutions (university hospitals, ministry of health and welfare, etc.) and pharmaceutical companies analyze medical data and use them in various fields such as drug development, infection, epidemiological studies, and health policy formulation [1], [2].

Diabetes mellitus is one of the major chronic diseases and top ten leading causes of death and the rates are increasing. It is associated with multiple health conditions such as heart and kidney failure. Diabetes mellitus has significantly impacts the quality of life (QoL) and cause health, social and economic burden to individuals, their families and community. However, these implications can significantly be reduced by controlling blood glucose level and practice active physical activities. Recent research indicates that using Internet of Things (IoT)-based diabetes health platform can help patients to self-care to the benefit of their health, both physical and mental. Patients who are proactive in the management of their chronic medical conditions can improve their health condition. As such, physician can use IoT-based and big-data diabetes health platform to facilitate patient compliance with physician recommendations and ultimately allow patients to manage and prevent their own chronic conditions on a routine basis in the outpatient condition. Diabetes mellitus is one of the most common chronic non-communicable diseases globally. The most prevalent form of diabetes is Type 2 diabetes, affecting about $91 \%$ of diabetes patients, while the remaining $9 \%$ mainly have Type 1 diabetes or gestational diabetes. Unhealthy dietary practices and lifestyle are the main cause of diabetes. Information and Communication Technology (ICT) interventions can reduce the risks of diabetesrelated complications by adopting healthy behavior. Cognitive computing and analytics along with the myriad of devices and sensors are available to help manage diabetes efficiently. In this study, Internet of Things (IoT) based big data platform will be developed and tested on 50 diabetes patients to improve behavioral management of Type 2 diabetes in an ethnically diverse population. Wearable devices can send data wirelessly to Bigdata platform based on MAPHIS [3] and healthcare professional will have access to the information. Data that is capture in the cloud computing can help patients and healthcare professional work together to manage the disease and make better decisions to improve quality of life in diabetes patients. 
In this study, we aim to develop a health care platform that receives diabetes information generated from various IoT based on remote inputs, stores, analyzes, processes and provides visualized information. The purpose of this study is to develop and test IoTbased diabetes health big-data platform for diabetes mellitus patients. To achieve this goal, we suggest the development result of service and contents oriented "An IoT-based diabetes health bigdata Offloading platform" that comprehensively manages healthcare products created in many IoT-based diabetes information to build a personal health management system. We also developed android 4.x-based application so that the health management service and contents provided by a third party can be checked with the client PC as well as health management service and contents offered by web-based client application and third party can be operated in the mobile environment such as smartphone or tablet.

The organization of this paper is as follows. Section 2 discusses the backgrounds and MAPHIS for our diabetes big-data platform. Section 3 put the implementation approaches, Section 4 discusses the issues and challenges in this area.

\section{Backgrounds and related works}

\subsection{Backgrounds}

The Apple's HealthKit [4] collects personal health information with external devices and applications as a platform that integrates and manages personal health information (PHI) to store and manage. The company establishes open healthcare ecosystem by attracting third party service providers in the healthcare industry. Google Fit [2], in the meantime, serves as a central storage that receives and shares health information and maintains more open platform than Apple's HealthKit. It also allows other external service providers to use the integrated PHI and access various information unlike the Apple's health application. Therefore, the other service providers can collect health data and develop application using them with Google Fit SDK. Google is making efforts to create an environment where they access much information and develop good applications focusing on use of personal fitness data. Google Fit, in particular, provides the foundation upon which application and devices meet, giving birth to lots of new health management services depending on combinations of existing applications and devices.

\subsection{MAPHIS}

As shown in figure 1, MAPHIS is a platform that complies with the ISO 11073 [5] DIM and HL7 protocols for interoperability of various healthcare devices and meets the ONE2M standard, the IOT international standard protocol for connectivity, as a practical support for SME healthcare service development.

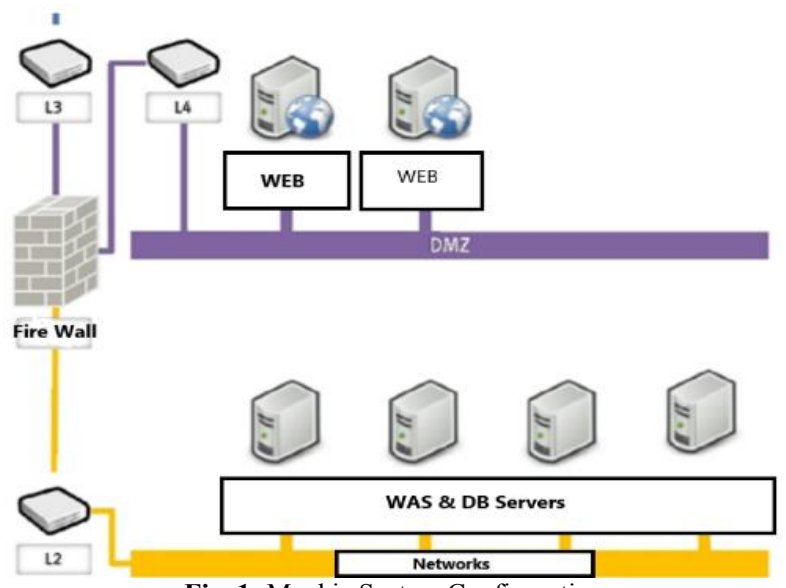

Fig. 1: Maphia System Configuration.

\section{An IoT-based diabetes health bigdata of- floading platform}

\subsection{System overview}

The purpose of this study is to develop and test IoT-based diabetes health big-data platform for diabetes mellitus patients. To achieve this goal, we suggest the development result of service and contents oriented "an IoT-based diabetes health big-data Offloading platform" that comprehensively manages healthcare products created in many IoT-based diabetes information to build a personal health management system as showing figure 2 .

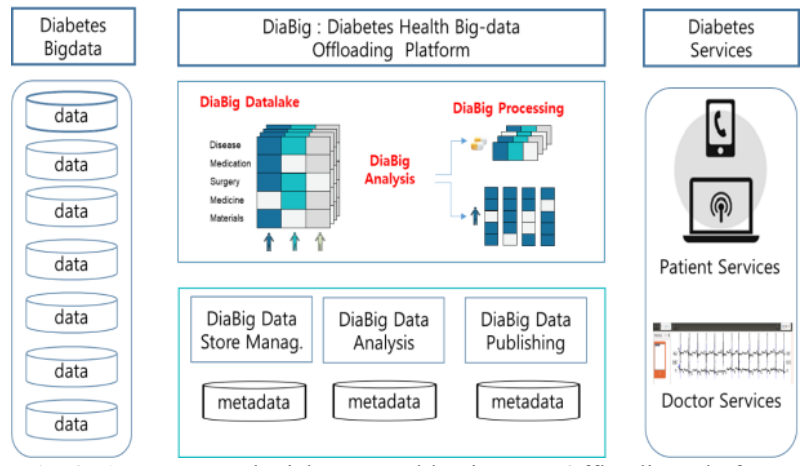

Fig. 2: An IoT-Based Diabetes Health Big-Data Offloading Platform.

An IoT-based big-data healthcare offloading platform proposed in this project is operated in close relation with platform operator, external service provider, client for health management and provision of health management device in and around the healthcare server. The health management platform server consists of platform administrator function, health device and linked API, PHI management website as well as health information big data analysis and inference engine that analyzes health information and provides service to the client [6]. It also uses HTML 5-based template and verified components to offer development productivity and fancy UX. The algorithm was developed that can effectively distribute the increasingly large volume of traffic along with increasing clients in the process of server establishment. When the client uses wearable devices, produced with external open platforms like Google Fit or Samsung SAMI, together with this platform, the app client software for web and mobile supported by the health management platform will enable the user to check his health condition and receive health management service and contents real time accordingly. In this study, Android 4.xbased application will be developed so that healthcare provider can assist in managing diabetes in patients by providing advice, services and contents through web-based client application. In addition, caregivers can contribute to diabetes management using mobile environment such as smartphone or tablet. The health management platform provides the means that can expand the function that the platform does not have in the form of open API (restful API) where third party solution or library can be linked to support API for the third party. It is composed to run as a Client-Server model. It sends the data requested by third party solution or library through API. The platform provides API in the form that enables calling through https and communicates by writing the full text with JSON as the standard. Since access is possible with various platforms, it takes the form that can process data requested from any platform. Lastly, API was designed to have usable levels to allow discrimination on functions depending on the level of user authority. The session information was made to minimize loading against the server by having the client manage it. Cache function was also installed on the server to respond to the substantial requests at optimal speed, thus overcoming the performance issue. The "IoT-based Diabetes health big-data analysis engine" embedded in the health management platform patterns and analyzes the feature of large volume of much health information generated or detected by the external health management device. After this, it induces useful infor- 
mation or rules and provides reliable health management information for the user based on objective data and description [7].

\subsection{Implementation stages}

At this $1^{\text {st }}$ stage, we first obtain laboratory data from two hospitals to analyze diabetes data on the big-data platform. It also secures diabetes sources from diverse devices based on IoT. To evaluate platform's functions and results, we apply the experimental data disclosed in National Diabetes Audit, Open data (https://data.gov.uk/dataset/national-diabetes-audit-opendata2010-2011) [8]. After verifying the function and results of DiaBig, we apply the field data collected from two actual hospitals. The raw source collected in the actual field is converted into a structured format in the two-step process.

At 2nd stage, Diabetes data collection and translation the structured, semi-structured, and unstructured diabetic big-data obtained in stage 1 are collected by dividing into internal data and external data and then reintegrated. The collection of internal data collects structured information by accessing the internal information held in the hospital or the database system being stored. The collection of external data acquires lab diabetes data such as MIT-BIT and acquires unstructured data through IoT based sensor. In the process of collecting big data, data warehouse, Big-data Lake, is saved by converting various source data into one common format through meaningful data Extraction, Transformation, and Loading(ETL) as shown in figure 3. As shown in figure 4, we use opensource OpenRefine [9](http://openrefine.org) to store large amounts of internal and external data in a single format. Currently, OpenRefine, which is installed and operated in software laboratory, is a neat tool for sorting out the nonrefined data. The basic function of OpenRefine is to fix common errors that often appear in generated datasets and to clean up unnecessary data contained in large amounts of data. It also adds functions such as error correction of text values, correction of inconsistent data, and data format conversion. In addition, it is possible to fix errors due to mis-entered entries in analyzing statistical data, or to make errors in the process of displaying different abbreviations and symbols among the same entries.

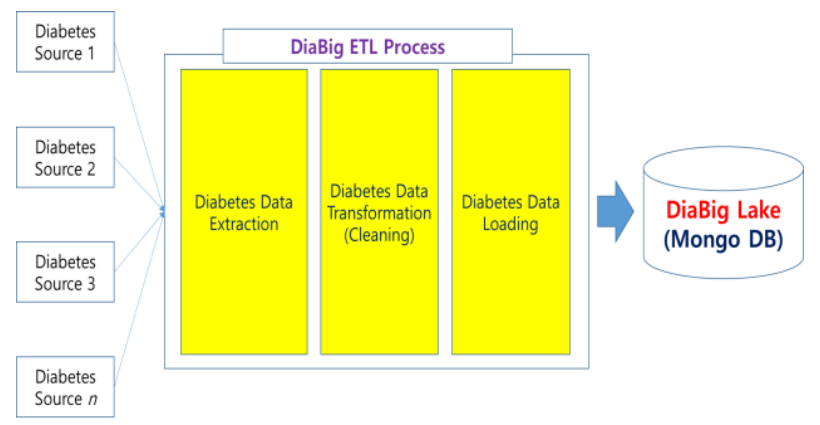

Fig. 3: ETL Processing for Diabetes Lake.

At $3^{\text {rd }}$ stage, to extract meaningful information from diabetes bigdata, efficient storage management technique is needed. Data storage management is to store data safely and efficiently for later use, and it requires a storage method capable of accommodating large volume, unstructured, and real-time attributes. It is important to have a technology capable of storing large amounts of data in the form of files and a technique of storing unstructured data in the form of standardized data. A distributed file system, NoSQL, a parallel DBMS, and a network configuration storage system are typical approaches. Among these approaches, this approach employs a NoSQL, MongoDB, methodology that simplifies the data model and considers all the DBMSs or data storage devices that do not use SQL. MongoDB is a database based on reliability and scalability, and it is open source developed for low management cost and commercial convenience in massive amounts of data. The unit of storage in MongoDB is the document, which is like the row in the RDBMS. Each document is collected in a collection similar to a database in an RDBMS, and each collection is man- aged in a database. MongoDB supports horizontal scaling with no loss of functionality due to automated sharding, aggregation operations such as range query, secondary index, sorting, and MapReduce of existing RDBMS. MongoDB allows distributed expansion using automated sharding. Sharding is the process of dividing the data and storing it in different servers, and it can manage and process more data by dividing the data into several servers. However, most DBMSs have difficulty in configuring sharding at application level. MongoDB solves this problem with automated sharding.

At 4th stage, Diabetes Data Processing. Big-data requires a technology that can integrate vast amounts of data, generation speed, and diversity of data types. As a data processing technology, Hadoop, R language and NoSQL. MapReduce technology integrates $<$ key, value $>$ input data segmentation and processing result integration technology, job scheduling technology, task distribution technology, and task re-execution technology to deal with a failure based on clustering system composed of general purpose server Distributed computing technology. At 5th stage, Most of the techniques used in the Big Data field are those already used in statistics, computer science, especially machine learning and data mining. The algorithms of these analytical techniques are modified or modified for large-scale data processing and applied to processing. classification, clustering, and machine learning techniques are applied to processing. The classification processing technique is a supervised learning method in which a training data group is learned in a previously known class group to find a data group to which the added data belongs. In this research study, we will adopt a KNN (K-Nearest Neighbor) algorithm [10]. The clustering processing technique is a learning method of grouping data having similar characteristics into group, and does not use the training data group unlike the classification processing technique. Therefore, it corresponds to the unsupervised method. Finally, machine learning processing technology is a model of human learning in the field of artificial intelligence, and it is trained to develop algorithms and techniques that enable computers to learn. 6th stage: Mobile Service and Visualization to Medical Professionals and Patients the DiaBig platform visualizes and delivers a variety of statistical information to medical professionals. Web and mobile information services for patients and caregivers will be developed.

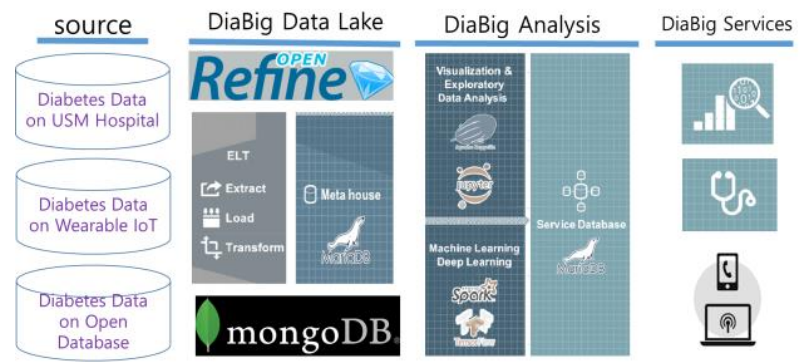

Fig. 4: Implementation Open Software Components.

Medical Professionals and Patients are offered simultaneous web and mobile services in offline and online environments. Especially, in online service process, it is possible to support real-time service considering support considering communication status. Medical Professionals and Patients are offered simultaneous web and mobile services in offline and online environments. Especially, in online service process, it is possible to support real-time service considering support considering communication status.

\section{Conclusion}

In this study, we suggested the development result of service and content-oriented healthcare Bigdata platform that comprehensively manages healthcare products created in many wearable IoT devices in a common ecosystem to build a personal health management system. The healthcare Bigdata platform suggested by the study is operated in close relation with platform operator, ex- 
ternal service provider, client for health management and provision of health management device in and around the offloading healthcare powerful server. In building healthcare big data platform, we adopt offloading technology for large data processing. Offloading technology is a technology that utilizes the computing resources of a remote, high performance server to reduce the processing speed of the mobile device and the burden of power consumption. Also, we propose a method to monitor the transmission data in real time and solve the transmission failure in order to smooth out the flow that can occur in the process of data transmission of a large capacity. The results of this study are verified by applying it to patients with diabetes or suspected cases. In order to increase the efficiency of real-time processing, we use off-loading technology to utilize big data related to diabetes generated from wearable IoT device. The results of this study will be used for telemedicine in two hospitals in Malaysia after various laboratory verification procedures.

\section{Acknowledgment}

This paper was supported by Joongbu University Research \& Development Fund, in 2018.

\section{References}

[1] J. Henriques, T. Rocha, S. Paredes, R. Cabiddu, D. Mendes, R. Couceiro, P. Carvalho(2015). ECG analysis to ol for heart failure management and cardiovascular risk assessment. Int'l Conf. Health Informatics and Medical Systems (HIMS2015), 195-200.

[2] A. Jonathan Garza, B. Sishir Subedi, C. Yuntian Zhan g and D Hong Lin (2015). A Web-Based System for EEG Data Visualization and Analysis. Int'l Conf. Health Informatics and Medical Systems (HIMS2015). 119-124

[3] MAPHIS (2017). Retrieved from http://http://www.maphis.or.kr/.

[4] Apple's HealthKit (2016). Retrieved fromhttps://developer.apple.com/healthkit.

[5] Health informatics-Personal health device communication (2018). Retrieved fromhttps://www.iso.org/standard/61897.html.

[6] Andrew V. Poliakov, Evan Albright, Kevin P. Hinsha w, David P. Corina, George Ojemann, Richard F. artin, James F. Brinkley(2005). Server-based Approach to Web Visualization of Integrated Three-dimensional Brain Imaging Data. Journal of the American Medical Informatics Association, 12(2), 140-151.

[7] Lourenco, A., Placido da Silva, H., Carreiras, C., Priscila Alves, A., L. N. Fred, A(2014). A web-based platform or biosignal visualization and annotation. Multimedia Tools and Applications, 70(1), $433-460$.

[8] National Diabetes Audit (2018), Retrieved from https://data.gov.uk/dataset/national-diabetes-audit-opendata-20102011.

[9] Open Refine92018). Retrieved fromhttp://openrefine.org.

[10] K-Nearest Neighbor algorithm. Retrieved from https://en.wikipedia.org/wiki/K-nearest_neighbors_algorithm. 